\title{
Polarization Control in Two-Color Above-Threshold Ionization of Atomic Helium
}

\author{
M. Meyer, ${ }^{1}$ D. Cubaynes, ${ }^{1}$ D. Glijer, ${ }^{1}$ J. Dardis, ${ }^{2}$ P. Hayden,${ }^{2}$ P. Hough,${ }^{2}$ V. Richardson, ${ }^{2}$ E. T. Kennedy, ${ }^{2}$ J. T. Costello, ${ }^{2}$ \\ P. Radcliffe, ${ }^{3}$ S. Düsterer, ${ }^{3}$ A. Azima,${ }^{3}$ W. B. Li,${ }^{3}$ H. Redlin, ${ }^{3}$ J. Feldhaus,${ }^{3}$ R. Taïeb, ${ }^{4}$ A. Maquet, ${ }^{4}$ \\ A. N. Grum-Grzhimailo, ${ }^{5}$ E. V. Gryzlova, ${ }^{5}$ and S. I. Strakhova ${ }^{5}$ \\ ${ }^{1}$ LIXAM, UMR 8624, CNRS-Université Paris Sud, Bâtiment 350, 91405 Orsay Cedex, France \\ ${ }^{2}$ National Center for Plasma Science and Technology and School of Physical Sciences, Dublin City University, Dublin, Ireland \\ ${ }^{3}$ HASYLAB at DESY, Notkestr. 85, D-22607 Hamburg, Germany \\ ${ }^{4}$ UPMC, Université Paris 06, CNRS, UMR 7614, LCPMR, 11 Rue Pierre et Marie Curie, 75231 Paris Cedex 05, France \\ ${ }^{5}$ Institute of Nuclear Physics, Moscow State University, Moscow 119991, Russia
}

(Received 27 June 2008; published 7 November 2008)

\begin{abstract}
Two-color multiphoton ionization of atomic helium was investigated by combining extreme ultraviolet (XUV) radiation from the Free Electron Laser in Hamburg with an intense synchronized optical laser. In the photoelectron spectrum, lines associated with direct ionization and above-threshold ionization show strong variations of their amplitudes as a function of both the intensity of the optical dressing field and the relative orientation of the linear polarization vectors of the two fields. The polarization dependence provides direct insight into the symmetry of the outgoing electrons in above-threshold ionization. In the high field regime, the monochromaticity of the XUV radiation enables the unperturbed observation of nonlinear processes in the optical field.
\end{abstract}

DOI: 10.1103/PhysRevLett.101.193002

PACS numbers: $32.80 . \mathrm{Fb}, 32.80 . \mathrm{Rm}$

Multiphoton single-color ionization in intense optical or infrared laser fields has been the subject of multiple experimental and theoretical studies for more than two decades and is by now a very well understood process (e.g., [1]). The extension of these studies to multiphoton absorption in the photoionization continuum was followed by the discovery that high order harmonics of the fundamental laser frequency are emitted in the extreme ultraviolet (XUV) when a strong femtosecond optical laser pulse interacts with a gas jet (e.g., [2,3]). The combination of different wavelengths, one in the XUV and the other in the visible or near infrared, opens new opportunities. It has recently permitted the investigation of above-threshold ionization (ATI) as the result of the combined interaction of both fields [4-6]. In this case the dominant contribution comes from processes in the course of which the emitted electron exchanges photons with the dressing laser field via stimulated emission (or absorption) resulting in a comb of sidebands disposed on both sides of the main photoelectron line.

Theoretical studies have established that the sideband intensity depends on the electron kinetic energy as well as on the strength and polarization state of the optical laser field [7]. Fitting theoretical profiles to the measured sideband signals should yield the main parameters which govern the photon-atom interaction in this regime. For example, changing the polarization of either of the radiation beams gives rise to "dichroic effects" in the photoelectron spectrum. It therefore opens the possibility to control the relative contributions of photoionization channels with different angular momenta.

This approach has been extensively used in studies of atomic ionization by weak monochromatic radiation from synchrotrons and continuous lasers, where at least one resonant intermediate state is involved, and the basic photon-electron interaction is completely dominated by this resonant excitation [8]. The use of high harmonic XUV sources to generate similar processes in the nonresonant continuum is complicated by very difficult analysis, since contributions from several harmonics and their mutual interferences have to be accounted for explicitly (e.g., [9-11]). As a result to date, it has not been possible to study the interference-free dependence of all photoelectron sidebands on the intensity and state of polarization of the dressing laser field.

In the present work, we used the unique properties of the Free electron LASer in Hamburg (FLASH) to perform for the first time this kind of study. Especially, the monochromaticity of FLASH enables us to scrutinize any two-color multiphoton ionization channel without additional overlapping contributions from neighboring harmonics. The observed sideband structures represent a direct testing ground for theory of the photon-atom interaction, since they are the direct signatures of the combined action of the XUV and optical fields. In particular, the results allow, in the low field regime, the determination of relative contributions of photoelectrons with different angular momenta, as well as, in the high field regime, the unperturbed analysis of higher order sidebands. This contrasts with previous experiments [10], where, for example, the study of secondorder sidebands was impeded by the presence of other harmonics. The data reported here, for both low and high dressing fields, provide benchmarking for dichroism studies with short wavelength free electron lasers and confirm that the current state of theory can account for all salient processes. 
Taking the $1 s$ ionization of atomic He by XUV photons as the primary example of our study (Fig. 1), at low optical laser intensities only one sideband is observed on each side of the main photoline. This sideband is produced by a twophoton process and the outgoing electron is described in terms of partial waves with $s$ and $d$ symmetry. For optical fields of higher intensities, multiple sidebands occur, and interferences between the different multiphoton pathways have to be taken into account leading to a more complex mixture of electrons with either even $(s, d, g, \ldots)$ or odd $(p, f, h, \ldots)$ symmetries.

The experiments have been performed using the twocolor pump-probe setup at FLASH [12]. The XUV FEL and the optical laser beams were introduced into the vacuum chamber in a collinear geometry and intersected an effusive gas jet within the acceptance volume of a magnetic bottle electron spectrometer (MBES). In order to ensure perfect time overlap between the pulses and to eliminate effects arising from the inherent time jitter of the FEL of about $500 \mathrm{fs}$ [13], the experiments were performed using picosecond optical lasers synchronized to the 10-20 fs FEL pulses [14]. The polarization of both lasers was practically linear $(\geq 99 \%)$ and the relative orientation between the polarization vectors could be changed by a rotatable halfwave plate in the optical path. The FEL was operated in single-bunch mode at a $5 \mathrm{~Hz}$ repetition rate and fundamental wavelengths of 25.5 and $13.7 \mathrm{~nm}$ (48.6 and $90.5 \mathrm{eV}$, respectively) with a mean pulse energy of about $20 \mu \mathrm{J}$. The optical field was produced either by a frequency doubled Nd:YLF laser $(523 \mathrm{~nm})$ delivering about $500 \mu \mathrm{J}$ pulses of 12 ps temporal width, or by an intense (up to $2 \mathrm{~mJ}, 800 \mathrm{~nm}, 4 \mathrm{ps}$ ) Ti:sapphire laser. The beam diameters of the FEL and the optical laser in the interaction volume were measured to be about 30 and $50 \mu \mathrm{m}$, respectively.

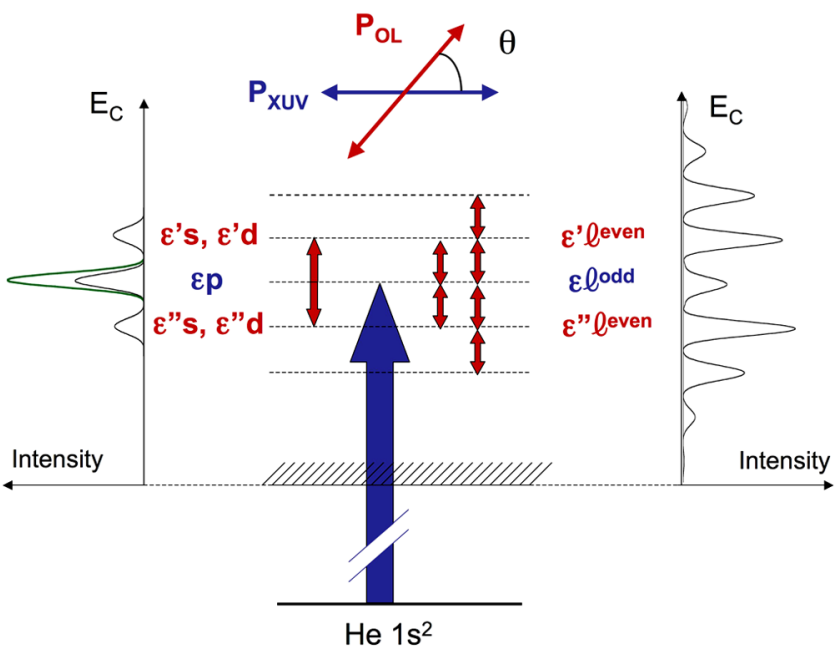

FIG. 1 (color online). Scheme of the two-color above threshold ionization in He atoms. The process and the resulting photoelectron spectra are shown for both low (left-hand side) and high (right-hand side) dressing fields. The relative orientation of the electric field vectors of the ionizing XUV radiation $\left(P_{\mathrm{XUV}}\right)$ and the optical dressing field $\left(P_{\mathrm{OL}}\right)$ is shown at the top.
The energy resolution in the electron spectra was mainly determined by the bandwidth of the FEL $(0.5 \%-1 \%)$ and the MBES (at best 1\%-2\% of the electron kinetic energies) [12].

A set of typical photoelectron spectra of He recorded for many single FEL pulses and for complete temporal and spatial overlap with the optical pulses $(800 \mathrm{~nm})$, is shown in Fig. 2. The spectra show one strong photoline attributed to the one-photon ionization of $\mathrm{He}$ by the FEL pulses leading to the $\mathrm{He}^{+} 1 s$ final state, and smaller sidebands on its high kinetic energy side, originating from two-color ATI. Only the high energy side is shown, since, in order to resolve the different lines, a retardation potential $(-60 \mathrm{~V})$ was applied to the entrance of the time-of-flight analyzer, which suppressed the low energy sidebands. Pronounced variations of the line amplitudes are observed as a function of the relative orientation of the linear polarization vectors of the XUV and the optical photons (Fig. 1). The sidebands are strongest when both polarization vectors are parallel and smallest when they are perpendicular. The growth of the sidebands is matched by a corresponding decrease in the main line; i.e., the total photoionization yield remains constant, as the optical field does not contribute to the primary ionization process.

The sensitivity of the electron emission to the polarization of both laser fields comes out more clearly, when the integrated intensity of the first sideband is displayed as a function of the relative orientation between the two linear polarization vectors (Fig. 3). Here a series of spectra was selected where only one sideband is observed on each side of the main line. This regime of low dressing field (about $8 \times 10^{10} \mathrm{~W} / \mathrm{cm}^{2}$ ) enables us to investigate directly the two-photon process. As the ionization starts from the $1 \mathrm{~s}$ shell, the variation of the sideband intensity is directly connected to the ratio between $s$ and $d$ electron waves,

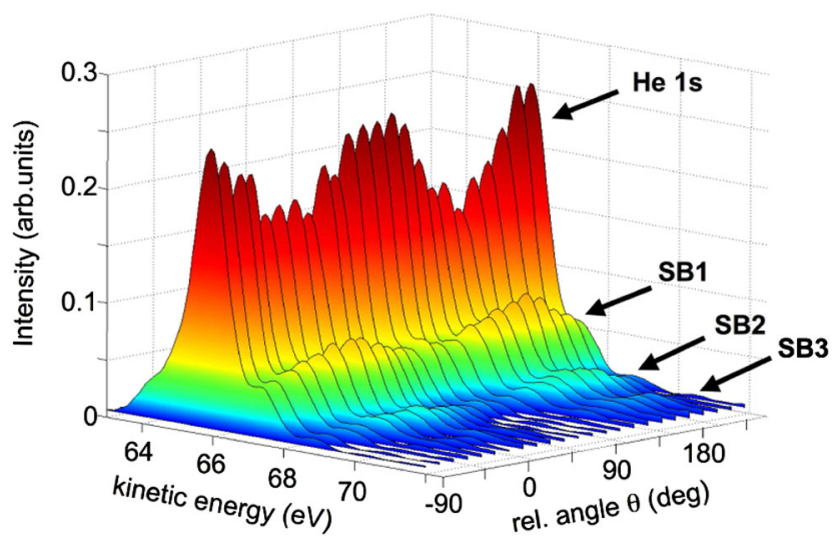

FIG. 2 (color online). Photoelectron spectra showing the He $1 s$ photoline and the high energy sidebands for high optical dressing fields $\left(\approx 6 \times 10^{11} \mathrm{~W} / \mathrm{cm}^{2}\right)$ upon photoionization at $90.5 \mathrm{eV}$. Spectra are presented for different relative orientations $\theta$ between the linear polarization vectors of the FEL and the $800 \mathrm{~nm}$ laser. 


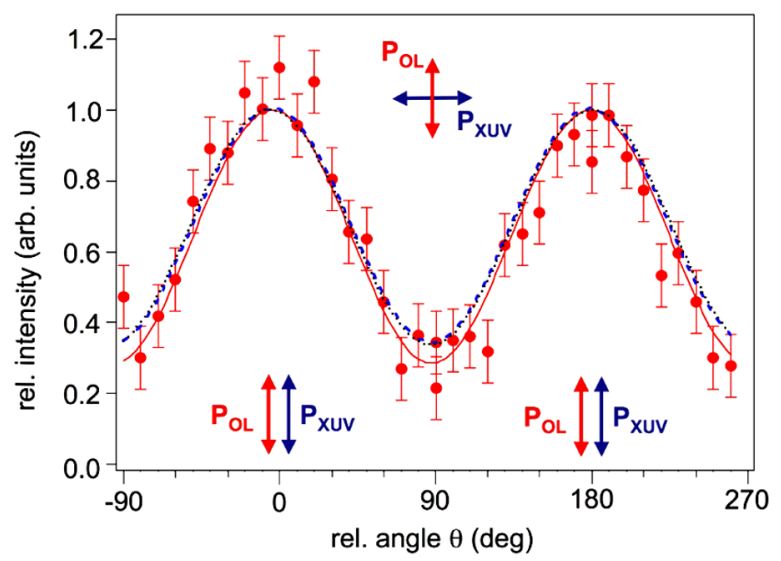

FIG. 3 (color online). Variation of the upper sideband yield in the low field regime $\left(\approx 8 \times 10^{10} \mathrm{~W} / \mathrm{cm}^{2}\right)$ for the two-photon ionization in the $\mathrm{He} 1 s$ shell at $13.7 \mathrm{~nm}$ as a function of the relative angle $\theta$ between the linear polarization vectors of the FEL and the $800 \mathrm{~nm}$ laser. The solid line denotes the fit to the experimental data (circles). The results of time-dependent second-order perturbation theory (dashed line) and soft-photon approximation (dotted line) are almost identical.

since their formation depends differently on the relative orientation of the optical and XUV polarization vectors.

A quantitative analysis of the partial wave contributions can be given by means of a time-dependent second-order perturbation calculation, which is adequate in this low intensity regime. The magnitude of the sidebands in the ionization of an $s$ electron in He can be described by

$$
\sigma(\theta) \propto 3 S_{2}+\left(5 S_{0}+S_{2}\right) \cos ^{2} \theta
$$

with $S_{i}=\left|T_{i}\right|^{2}$, where $T_{i}(i=0,2)$ are second-order radial integrals associated with the emission of $s$ and $d$ electrons, respectively [15]. Numerical calculations were performed using the experimental conditions at FLASH and taking a "single active electron" model with a local potential for He.

Also, the "soft-photon" approach can be used instead to account for the departure from the lowest-order perturbation contribution and for higher sidebands [7]. Within the framework of this approximation, the differential cross section for the exchange of $n$ laser photons of frequency $\omega_{L}$ together with the absorption of the XUV photon $\omega_{X}$, is given by

$$
\frac{d \sigma^{(n)}}{d \Omega}=J_{n}^{2}\left(\vec{\alpha}_{0} \cdot \vec{k}_{n}\right) \frac{d \bar{\sigma}}{d \Omega}
$$

where $k_{n}=\sqrt{2\left(E_{1 s^{2}}+\omega_{X}+n \omega_{L}\right)}$ is the shifted wave number of the ejected electron, $\vec{\alpha}_{0}=\vec{F}_{0 L} / \omega_{L}^{2}$ with $\vec{F}_{0 L}$ the optical laser electric field and $d \bar{\sigma} / d \Omega$ the one-photon $\left(\omega_{X}\right)$ cross section. For ionization of a He $1 s$ electron, the cross section $\sigma^{(n)}$ after integration over the electron solid angle $\Omega \equiv(\eta, \phi)$ is given by

$$
\sigma^{(n)} \propto a_{n}+b_{n} \cos ^{2} \theta,
$$

where $\theta$ denotes the angle between the polarization directions of the two fields and the coefficients $a_{n}$ and $b_{n}$ are radial components containing information about the dynamics of the process. At low laser intensity, i.e., $\alpha_{0} k_{n} \ll$ 1 , the Bessel function, $J_{n}$, is proportional to $\left(\alpha_{0} k_{n} \cos \eta\right)^{n}$, which gives $b_{n} / a_{n}=2 n$, leading to a contrast of $\sigma_{\max }^{(n)} / \sigma_{\min }^{(n)}=2 n+1$ in the $\theta$ dependence of $\sigma^{(n)}$.

Theoretical results in the low field regime show a maximum of the ionization signal at parallel and a minimum at perpendicular polarizations (Fig. 3). They are in excellent agreement with the experimental data and the differences between the two approaches are hardly visible on the scale of presentation. The measured contrast $\sigma_{\max } / \sigma_{\min }$ of $3.4 \pm$ 0.5 is consistent with the theoretical value of 3 and leads to an experimental ratio $S_{0}: S_{2}=1.25 \pm 0.3$, in good agreement with the theoretical values of $S_{0}: S_{2}=0.98$ and 1.0 obtained within the second-order perturbation calculation and the soft-photon approach, respectively. For the case of parallel polarizations $\left(\theta=0^{\circ}\right)$, Eq. (3) shows that the experimental value $S_{0}: S_{2}$ results in a ratio of 1.5 for the relative yields of $s\left(5 S_{0}\right)$ and $d\left(4 S_{2}\right)$ electrons.

The contributions from $s$ and $d$ electrons in the sideband deviate strongly from the propensity rules predicting that, upon absorption of one photon, transitions related to an increase of the angular momentum $(\ell \rightarrow \ell+1)$ should be favored [16]. Here, the determined ratio differs from the value obtained in studies on the two-color ionization via discrete intermediate states, $\mathrm{He}^{*} 1 \operatorname{snp}{ }^{1} P(n=2,3)$, where a more than 10 times higher cross section for $d$ than for $s$ electrons was determined [17]. For resonant ionization, the overlap between the discrete $n p$ and continuum $s$ or $d$ electron wave functions governs the branching ratio. Close to the ionization threshold for $\mathrm{He}$, due to the centrifugal barrier, the first lobe of the $d$ wave overlaps considerably the lobe of the $n p(n=2,3)$ wave function. In contrast, the first oscillation of the $s$ wave is much closer to the nucleus, leading to compensation of positive and negative contributions to the transition matrix elements. Near the threshold, the $d$ wave dominates the resonant two-color ionization. For the ATI case, the strengths of the $p \rightarrow s$ and $p \rightarrow d$ transitions are mainly determined by the overlap of the oscillatory asymptotics of the coupled continuum states, roughly by the squared cosine of their scattering phase difference [18]. This quantity varies smoothly with the energy of the coupled continuum states and accords preference to the $s$ channel for $\mathrm{He}$ at the experimental energies.

The strong contribution of the $s$ wave in the two-photon ionization process far above threshold is also present at lower XUV photon energies. The experiments using the FEL at $25.5 \mathrm{~nm}(48.6 \mathrm{eV})$ in combination with the $(523 \mathrm{~nm})$ optical laser for the dressing field resulted in a ratio $S_{0}: S_{2} \approx 0.9 \pm 0.3$, i.e., smaller than the previous value. This underlines the general tendency that, at lower excitation energies, the contribution of the $d$ wave increases, in better accordance with the propensity rules. This experi- 


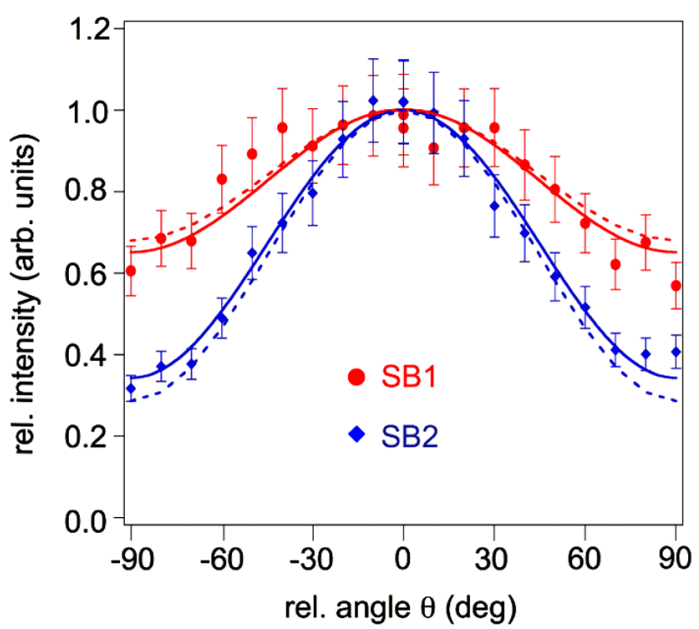

FIG. 4 (color online). Variation of the sideband yield in the high field regime $\left(\approx 6 \times 10^{11} \mathrm{~W} / \mathrm{cm}^{2}\right)$ for the first (circles) and second (diamonds) sideband of the He $1 s$ ionization at $13.7 \mathrm{~nm}$ as a function of the relative angle $\theta$ between the linear polarization vectors of the ionizing FEL and the $800 \mathrm{~nm}$ dressing fields. The solid lines are a fit to the experimental data, the dashed lines are the results of the soft-photon approximation.

mental ratio is in good agreement with the value of 0.85 obtained in the simulation.

With a stronger optical dressing field $\left(\geq 10^{11} \mathrm{~W} / \mathrm{cm}^{2}\right)$, more than one sideband is observed (cf. Fig. 2). This situation cannot be treated through the second-order perturbation theory, while the soft-photon approximation is still appropriate to calculate the strengths of the higher sidebands and their polarization dependence.

In Fig. 4, the variations of the first two sideband yields are given for photoionization at $13.7 \mathrm{~nm}$ and a higher dressing field $\left(800 \mathrm{~nm}, 6 \times 10^{11} \mathrm{~W} / \mathrm{cm}^{2}\right)$. The amplitude of oscillation of the first sideband is less pronounced than for the case where only one sideband is observed (cf. Fig. 3). The contrast $\sigma_{\max }^{(1)} / \sigma_{\min }^{(1)}$ is now only $1.5 \pm$ 0.3 compared to $3.4 \pm 0.5$ in the low field regime. Once again, the soft-photon approach describes satisfactorily the main features of the experiment, giving a ratio of 1.47.

The reduced contrast in the angular distribution can be explained when performing a development of the Bessel function in Eq. (1) for larger value of its argument. It shows that the ratio $b_{1} / a_{1}$ becomes smaller when the intensity of the dressing field is increased. This is caused by contributions from more quantum paths (associated with the exchange of more photons) leading to the same final state, as compared to the low field regime (see Fig. 1). Then, in a given sideband, higher angular momenta are populated. In the case of the first sideband (SB1), this results in a destructive interference when the two fields are parallel, thus diminishing the contrast $\sigma_{\max }^{(1)} / \sigma_{\min }^{(1)}$. This is reminiscent to what was observed when combining XUV harmonics with an IR laser field: the contribution of higher angular momenta resulted in the decrease of the relative maxima in the angular distributions [19]. This trend is confirmed in the case of the second sideband $(n=2)$. While the contrast $\sigma_{\max }^{(2)} / \sigma_{\min }^{(2)}$ is significantly larger than for the first sideband ( $\approx 3.3$ in the experiment and $\approx 3.5$ in the calculations), it does not reach the limiting value of $2 n+1=5$, that is predicted by the soft-photon approximation.

In conclusion, we have shown that the investigation of the polarization dependence of the sidebands in two-color ATI provides detailed insight into the dynamics of this class of process. The monochromaticity of the XUV radiation from FLASH enables us to study the ATI process free from disturbing interference processes. Good overall agreement is found between experiment and theoretical results, giving confidence that our next-step studies will even allow us to extract the phases of the partial waves, i.e., make feasible a complete description of the two-color ionization process.

We greatly appreciate the work of the scientific and technical team at FLASH, in particular, the machine operators and run coordinators. Support from the EU RTDproject X-Ray FEL Pump-Probe HRPI-CT-1999-50009, from Science Foundation Ireland (Frontiers), IRCSET and the HEA PRTLI 4 Scheme, and from the FrenchIreland ULYSSES program are acknowledged.

[1] L.F. Di Mauro and P. Agostini in Advances in Atomic, Molecular, and Optical Physics, edited by B. Bederson and H. Walther (Academic, New York, 1995), Vol. 35, p. 79.

[2] P. Salières, A. L'Huillier, P. Antoine, and M. Lewenstein in Advances in Atomic, Molecular, and Optical Physics, edited by B. Bederson and $\mathrm{H}$. Walther (Academic, New York, 1999), Vol. 41, p. 83.

[3] J. G. Eden, Prog. Quantum Electron. 28, 197 (2004).

[4] T.E. Glover, R. W. Schoenlein, A.H. Chin, and C. V. Shank, Phys. Rev. Lett. 76, 2468 (1996).

[5] J. M. Schins et al., Phys. Rev. A 52, 1272 (1995).

[6] E. S. Toma et al., Phys. Rev. A 62, 061801 (2000).

[7] A. Maquet and R. Taïeb, J. Mod. Opt. 54, 1847 (2007).

[8] S. Baier, A. N. Grum-Grzhimailo, and N. M. Kabachnik, J. Phys. B 27, 3363 (1994).

[9] V. Véniard, R. Taïeb, and A. Maquet, Phys. Rev. A 54, 721 (1996).

[10] P. O'Keeffe et al., Phys. Rev. A 69, 051401(R) (2004).

[11] O. Guyétand et al., J. Phys. B 38, L357 (2005).

[12] P. Radcliffe et al., Nucl. Instrum. Methods Phys. Res., Sect. A 583, 516 (2007).

[13] P. Radcliffe et al., Appl. Phys. Lett. 90, 131108 (2007).

[14] W. Ackermann et al., Nat. Photon. 1, 336 (2007).

[15] R. Taïeb et al., Phys. Rev. A 62, 013402 (2000).

[16] U. Fano, Phys. Rev. A 32, 617 (1985).

[17] A. Johansson et al., Eur. Phys. J. D 22, 3 (2003).

[18] T. Mercouris et al., J. Phys. B 29, L13 (1996).

[19] O. Guyétand et al., J. Phys. B 41, 051002 (2008). 